\title{
The impact of human metabolism on the bioactivity of anthocyanins
}

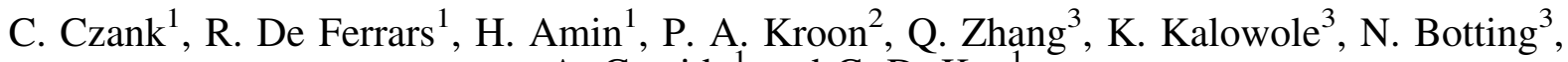 \\ A. Cassidy $^{1}$ and C. D. Kay ${ }^{1}$ \\ ${ }^{1}$ Department of Nutrition, Norwich Medical School, University of East Anglia, Norwich, NR47TJ, \\ ${ }^{2}$ Institute of Food Research, Norwich, NR47UA and ${ }^{3}$ School of Chemistry, University of St Andrews, KY1 69AJ, \\ Scotland, UK
}

Diet derived anthocyanins $(\mathrm{ACN})$ such as cyanidin-3-glucoside $(\mathrm{C} 3 \mathrm{G})$ are believed to reduce the risk of cardiovascular disease $(\mathrm{CVD})^{(1,2)}$ despite their apparent low bioavailability ${ }^{(3)}$. However, given that ACN are likely to degrade following ingestion, their degradation products and metabolites are likely to contribute to their bioactivity ${ }^{(4)}$.

In our recently completed human feeding study in which stable isotope labelled C3G was fed to healthy male participants (500 mg bolus of ${ }^{13} \mathrm{C}_{5}-\mathrm{C} 3 \mathrm{G} ; n=8$ ), a total of 22 metabolites were identified. This included protocatachuic acid (PCA) which had a $\mathrm{C}_{\mathrm{Max}}$ of $0.23 \pm 0.12 \mu \mathrm{M}$ and its methylated derivative vanillic acid (VA) which had a $\mathrm{C}_{\mathrm{Max}}$ of $0.66 \pm 0.41 \mu \mathrm{M}$. We explored the bioactivity of these metabolites on vascular health by measuring the effects on mediators of nitric oxide bioavailability including endothelial nitric oxide synthase (eNOS), NADPH oxidase (NOX4) and superoxide. Protein expression of eNOS was measured by ELISA and NOX4 by western blotting, while superoxide was measured indirectly via ferrocytochrome $\mathrm{C}$ oxidation $\left(\mathrm{A}_{550}\right)$ in cultured human umbilical cord vascular endothelial cells treated with either $0.1,1$ or $10 \mu \mathrm{M}$ of $\mathrm{C} 3 \mathrm{G}$, PCA or VA.

\begin{tabular}{|c|c|c|c|c|c|c|c|}
\hline \multirow[b]{2}{*}{ Compound } & \multirow[b]{2}{*}{ Concentration } & \multicolumn{2}{|c|}{ eNOS protein } & \multicolumn{2}{|c|}{ NOX4 Protein } & \multicolumn{2}{|c|}{ Superoxide } \\
\hline & & Mean & SD & Mean & $\mathrm{SD}$ & Mean & SD \\
\hline \multirow[t]{3}{*}{ Cyanidin-3 glucoside } & $0.1 \mu \mathrm{M}$ & 63 & 27 & 106 & 27 & $85^{*}$ & 12 \\
\hline & $1 \mu \mathrm{M}$ & $337 *$ & 11 & 65 & 26 & $82 *$ & 3 \\
\hline & $10 \mu \mathrm{M}$ & 138 & 20 & 64 & 50 & 113 & 8 \\
\hline \multirow[t]{3}{*}{ Protocatachuic acid } & $0.1 \mu \mathrm{M}$ & $58 *$ & 3 & 79 & 45 & 93 & 30 \\
\hline & $1 \mu \mathrm{M}$ & $49 *$ & 27 & $40 *$ & 9 & $48 *$ & 21 \\
\hline & $10 \mu \mathrm{M}$ & $44^{*}$ & 43 & 20.1 & 17.1 & $32 *$ & 21 \\
\hline \multirow[t]{3}{*}{ Vanillic acid } & $0.1 \mu \mathrm{M}$ & $357 *$ & 2 & 92.3 & 10.6 & 56 & 34 \\
\hline & $1 \mu \mathrm{M}$ & 82 & 20 & 80.2 & 14.8 & 119 & 25 \\
\hline & $10 \mu \mathrm{M}$ & $236^{*}$ & 35 & 35 & 29 & 25 & 9 \\
\hline
\end{tabular}

Values are given as percentage relative to control and are the mean of 3 independent experiments $* P<0.05$ (ANOVA with Tukey Kramer test, $n=3$ ).

C3G significantly increased eNOS at a concentration of $10 \mu \mathrm{M}$ and significantly decreased superoxide at 0.1 and $1 \mu \mathrm{M}$ but had no effect on NOX4 protein expression. PCA signficantly decreased eNOS protein at all concentrations tested, reduced NOX4 protein at $1 \mu \mathrm{M}$ and decreased superoxide at 0.1 and $10 \mu \mathrm{M}$. VA significantly increased eNOS at 0.1 and $10 \mu \mathrm{M}$, but did not affect NOX4 expression or superoxide production. These studies provide early evidence that metabolites of anthocyanins may exhibit greater bioactivity than the parent molecule. In addition, these activities are apparent at relatively low and dietary achievable concentrations.

This work was supported by Biotechnology and Biological Sciences Research Council Diet and Health Industry Club.

1. Cassidy A, O’Reilly A, Kay C et al. (2011) Am Soc Nutrition 93, 338-47.

2. Hooper L, Kroon PA, Rimm EB et al. (2008) Am Soc Nutrition 88, 38-46.

3. Kay CD. (2006) Nutr Res Rev 19, 137-46.

4. Kay CD, Kroon PA, Cassidy A (2009) Mol Nutr Food Res 53, S92-S101. 